\title{
CTLA4-Ig in B7-1-positive diabetic and non-diabetic kidney disease
}

\author{
Roberto Bassi $^{1,2}$ - Alessia Fornoni ${ }^{3}$ - Alessandro Doria ${ }^{4}$ Paolo Fiorina ${ }^{1,2}$
}

Received: 14 July 2015 / Accepted: 19 August 2015 /Published online: 26 September 2015

(C) Springer-Verlag Berlin Heidelberg 2015

\begin{abstract}
Diabetic kidney disease (DKD) is the leading cause of end-stage renal disease in the Western world. Standard treatments have ultimately proven ineffective in blocking DKD progression, thus necessitating the design of new therapies to complement glycaemic and blood pressure control. High glucose levels upregulate the immune-related molecule B7-1 in podocytes, and such an event may play a relevant role in DKD onset, suggesting that B7-1 is a suitable therapeutic target for DKD. CTLA4-Ig is a clinically available fusion protein, approved for the treatment of some autoimmune diseases, which binds B7-1 and blocks its signalling. We have previously demonstrated that CTLA4-Ig restores the physiological structure and cellular motility of podocytes challenged with high glucose in vitro and abrogates the onset of proteinuria in murine models of DKD in vivo. Notably, these beneficial effects occurred independently of any systemic immunological effects of CTLA4-Ig. While the expression of B7-1 on podocytes raises questions regarding the very nature of the podocyte as we know it, the preliminary positive effect of CTLA4-Ig on proteinuria in preclinical models and the
\end{abstract}

Paolo Fiorina

paolo.fiorina@childrens.harvard.edu

1 Division of Nephrology, Boston Children's Hospital, Harvard Medical School, 300 Longwood Ave., Enders Building, Boston, MA 02115, USA

2 Department of Transplant Medicine, IRCCS Ospedale San Raffaele, Milan, Italy

3 Peggy and Harold Katz Family Drug Discovery Center, Division of Nephrology and Hypertension, University of Miami Miller School of Medicine, Miami, FL, USA

4 Section on Genetics and Epidemiology, Joslin Diabetes Center, Boston, MA, USA evidence of B7-1 expression in kidney biopsies of diabetic individuals suggest a potential novel indication for CTLA4Ig in DKD. Nonetheless, recent reports of problems with detecting podocyte B7-1 and of inconsistent therapeutic efficacy of CTLA4-Ig in proteinuric patients highlight the necessity to establish uniformly accepted protocols for the detection of B7-1 and underline the need for randomised trials with CTLA4-Ig in kidney diseases.

Keywords B7-1 · CD80 · CTLA4-Ig · Diabetic kidney disease $\cdot$ Podocytes $\cdot$ Review
Abbreviations
DKD Diabetic kidney disease
ESRD End-stage renal disease
FSGS Focal segmental glomerulosclerosis
LPS Lipopolysaccharide
MCD Minimal change disease
TLR4 Toll-like receptor 4

\section{Diabetic kidney disease}

Diabetic kidney disease (DKD) affects nearly $40 \%$ of patients with type 1 and type 2 diabetes [1,2] and accounts for $44 \%$ of end-stage renal disease (ESRD) cases in the USA [3]. DKD is associated with increased urinary albumin excretion, progressive decline of GFR and increased systemic blood pressure, ultimately leading to kidney failure [4]. Changes in kidney structure and function begin with glomerular hyperfiltration, followed by hypertrophy, podocytopenia, expansion of mesangial components and thickening of the basement membrane, which eventually progress to classical 
glomerulosclerosis and tubulo-interstitial alterations [4]. These pathological changes have been correlated in the past with the clinical progression of microalbuminuria (AER $>30 \mathrm{mg} / 24 \mathrm{~h}$ and $\leq 300 \mathrm{mg} / 24 \mathrm{~h}$ ) to macroalbuminuria $($ AER $>300 \mathrm{mg} / 24 \mathrm{~h}$ ) [5]; however, recent data suggest that not all diabetic patients advance to overt proteinuria - with some even regressing to normoalbuminuria $[6,7]$ - and that GFR decline may occur in the absence of albuminuria [8]. Nonetheless, albuminuria remains a strong risk factor for cardiovascular mortality [9], and proteinuric patients are likely to die of a cardiovascular event rather than progressing to ESRD and/or undergoing dialysis or a renal transplant procedure [10], thus suggesting that both GFR-sparing and AERreducing strategies should be taken into account for the treatment of kidney complications in diabetic patients [11]. The current therapeutic paradigm for the primary prevention of DKD focuses mainly on the strict management of hyperglycaemia and targeting the renin-angiotensin-aldosterone system when hypertension is present. Strict blood glucose control $\left(\mathrm{HbA}_{1 \mathrm{c}}<7 \% ;<53 \mathrm{mmol} / \mathrm{mol}\right)$ [12] plays a pivotal role in reducing the risk of DKD in both type 1 and type 2 diabetic patients [13]. Notably, a recent Cochrane metaanalysis confirmed the effectiveness of tight glucose control on primary prevention of microvascular complications; however, the effects on the progression of DKD seem to lessen once these complications have become manifest [14]. Much evidence suggests that patients with DKD greatly benefit from treatment with angiotensin-converting enzyme inhibitors and angiotensin receptor blockers [15]. The renoprotective activity of these drugs not only relies on their reducing intraglomerular pressure [16], but also on inhibiting the induction of proinflammatory and profibrotic mediators, which play a critical role in further jeopardising renal function [16]. Unfortunately, although these treatments can delay the onset of DKD, they cannot ultimately prevent it.

\section{Podocytes as immune-like cells}

Podocytes are a subset of terminally differentiated epithelial cells located within the kidney glomerulus that build a fine cellular and multiproteic filter through which plasma can percolate to produce a virtually protein-free milieu $[17,18]$. Apart from being essential structural components of the renal filtration barrier, recent data suggest that podocytes may also be regarded as immune-like cells of the glomerular microenvironment. Indeed, under inflammatory conditions, podocytes exhibit increased expression of MHC class I and II molecules and are also capable of removing immunoglobulins and immune complexes from the glomerular membrane [19]. Furthermore, podocytes can acquire and process antigens to stimulate specific $\mathrm{CD}^{+}$and $\mathrm{CD} 8^{+} \mathrm{T}$ cell responses, mimicking some of the functions of dendritic cells or macrophages of hematopoietic origin [19]. Podocytes have also been shown to constitutively express Toll-like receptor 4 (TLR4), and stimulation with TLR4-specific ligands results in potent induction of the costimulatory molecule B7-1 and a wide array of proinflammatory and profibrotic factors $[20,21]$. In this respect, stimuli of a broad nature have been linked to modification of the podocyte secretome in vitro and in vivo, leading to enhanced secretion of cytokines/chemokines (e.g. IL-1, monocyte chemoattractant protein-1 and IL-8) [22-24], which may favour the recruitment of inflammatory cells [25] or alter podocytes in an auto-/paracrine fashion [24]. Podocytes also express elements of the complement system in normal (e.g. complement receptor 1 [CR1]) [26] and pathological (e.g. C5a receptor) [27] conditions. Finally, podocytes can express CD40, and autoantibodies against this protein have been shown to predict proteinuria onset in focal segmental glomerulosclerosis (FSGS) [28]. This newly suggested nature of podocytes as immune-like cells, although far from being fully demonstrated, could provide an entirely new spectrum of therapeutic targets to enrich classical strategies for the preservation of kidney function in multiple diseases [29].

\section{Podocytes and B7-1}

B7-1 (also termed CD80) is a transmembrane protein usually expressed by antigen-presenting cells which, upon engagement of CD28 expressed by $\mathrm{T}$ cells, provides a positive costimulatory signal in the context of antigen recognition, leading to $\mathrm{T}$ cell activation, proliferation and survival [30, 31] (Fig. 1a). In 2004 Reiser et al reported that in vitro exposure of podocytes to lipopolysaccharide (LPS), a component of the outer membrane of Gram-negative bacteria that elicits strong immune responses, could unexpectedly result in the upregulation of B7-1, with alterations of podocyte shape and mild transient proteinuria when administered in vivo in a murine model of nephrotic syndrome [21]. More recently, other stress factors have been linked to podocyte B7-1 induction. For example, albumin has been shown to induce B7-1 expression via a cyclooxygenase-2-mediated mechanism, causing podocyte damage in vitro and albuminuria in vivo [32], while plasminogen activator inhibitor type 1 directly injures the podocyte by increasing desmin and B7-1 mRNA expression [33]. Hypoxia inducible factor-1 has also been reported to cause cytoskeleton rearrangement and increased podocyte motility by interacting with B7-1 [34] and, recently, polyinosinic-polycytidylic acid (polyIC) treatment in mice has been shown to result in podocyte B7-1 upregulation and the onset of proteinuria [35]. Interestingly, despite some contrasting findings reported by Ishimoto et al, indicating a certain degree of urinary protein excretion in B7-1 knockout mice (probably due to the persistence of the intracellular portion of B7-1 in the knockout mice) [35], our group and others [21] 
a

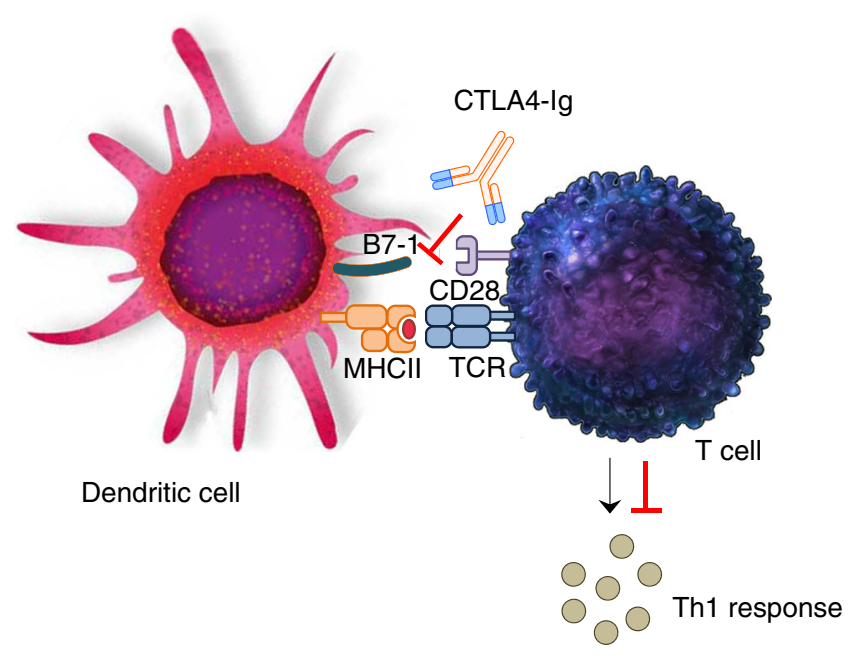

b

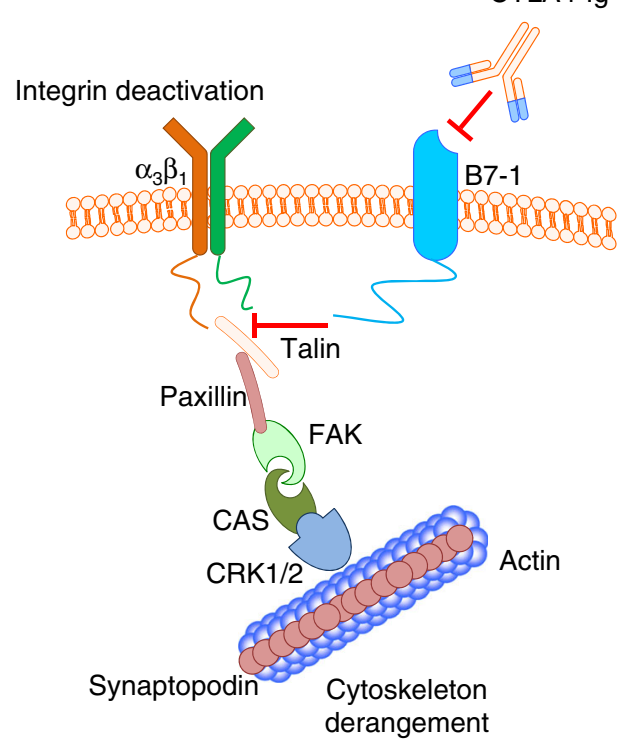

Immunological synapsis

Fig. 1 (a) Simplified scheme of the immunological synapsis created by the interaction of dendritic cells and T cells. By antagonising CD28 binding to B7-1, CTLA4-Ig dampens T cell activation and survival, thus exerting immunomodulatory functions. (b) Hypothetical mechanism of B7-1 cytoplasmic tail interference with the anchoring of $\alpha_{3} \beta_{1}$ integrin to

have demonstrated that B7-1 knockout mice are protected from LPS- or hyperglycaemia-induced proteinuria, and that mice without $\mathrm{B}$ and $\mathrm{T}$ cells fail to develop proteinuria after podocyte B7-1 inhibition with CTLA4-Ig, a clinical-grade fusion protein. The protein, which consists of the extracellular domain of human CTLA4 linked to the modified Fc portion of the immunoglobulin $\mathrm{G}_{1}$, selectively binds B7-1 and is used as an immunomodulatory agent in a variety of autoimmune conditions (Fig. 1a and Table 1) [36]. The efficacy of CTLA4-Ig in the absence of a competent immune system suggests that a systemic response could not explain the anti-proteinuric effect of B7-1 targeting [21]. More recently, Mundel and colleagues proposed a potential mechanism of B7-1-mediated podocyte the cytoskeletal complex of the podocyte. By binding to the extracellular portion of B7-1, CTLA4-Ig inhibits this disease-generating process, restoring the normal podocyte physiological structure and function. CAS, CRK-associated substrate; FAK, focal adhesion kinase; MHCII, MHC class II molecule; TCR, T cell receptor; Th1, T helper type 1

damage, hypothesising that the upregulation of extracellular B7-1 is intimately connected with the state of activation of $\alpha_{3} \beta_{1}$ integrin [37] (Fig. 1b). Podocyte foot processes are longitudinally anchored to the glomerular basement membrane predominantly via $\alpha_{3} \beta_{1}$ integrin, an obligate heterodimer ( $\alpha / \beta$ subunits) that, depending on mutual interactions between the integrin cytoplasmic tails and elements of podocyte cytoskeleton, oscillates between low or high affinity states for specific amino acid motifs of the glomerular basal membrane $[37,38]$. The findings to date suggest that podocyte B7-1 would negatively modulate $\alpha_{3} \beta_{1}$ integrin through direct interaction with integrin tails and mechanistically compete with talin [39] (a cytoskeleton-associated protein with important
Table 1 Tested applications of CTLA4-Ig in the clinical setting to date

\begin{tabular}{lllll}
\hline Action & Disease & Phase & Clinicaltrials.gov & Efficacy (Y/N) \\
\hline Immunomodulation & Rheumatoid arthritis & 3 & NCT00559585 & $\mathrm{Y}$ \\
& Recent-onset T1D & 2 & NCT00505375 & Y/N \\
& Ulcerative colitis & 3 & NCT00410410 & $\mathrm{N}$ \\
& SLE & 2 & NCT00119678 & $\mathrm{N}$ \\
& Lupus nephritis & 1 & NCT00705367 & $\mathrm{N}$ \\
& Wegener's granulomatosis & 2 & NCT00468208 & $\mathrm{Y}$ \\
Anti-proteinuric & Atopic asthma & 2 & NCT00784459 & $\mathrm{N}$ \\
& FSGS & - & - & $\mathrm{Y} / ?$ \\
& DKD & - & - & - \\
\hline
\end{tabular}

FSGS, focal segmental glomerulosclerosis; SLE, systemic lupus erythematosus; T1D, type 1 diabetes 
bridge functions between the cell membrane and the cytoskeleton) $[18,37]$ (Fig. 1b). However, additional roles for B7-1 in podocytes cannot be excluded. The cytoplasmic tail of B7-1 has been demonstrated to be necessary and sufficient to block $\alpha_{3} \beta_{1}$ integrin activation, thus causing morphological and functional alterations in the podocyte $[18,37]$. In vitro treatment of podocytes with CTLA4-Ig inhibited the effect of B7-1 on $\alpha_{3} \beta_{1}$ integrin, preventing disease-associated podocyte migration, which is thought to be a primary cause of glomerular barrier disruption and a surrogate for proteinuria onset in vivo (Fig. 1b) $[18,37]$. Importantly, in the clinical setting, CTLA4Ig treatment induced partial or complete remission of proteinuria in four patients with rituximab-resistant secondary FSGS [40] (treated with a single $[n=2]$ or double $[n=2]$ injection at a dose of $10 \mathrm{mg} / \mathrm{kg}$ ), and in one patient with steroid-resistant primary FSGS [37] (treated with multiple monthly doses of $10 \mathrm{mg} / \mathrm{kg}$ ), thus suggesting that CTLA4-Ig may have antiproteinuric effects. Upregulation of podocyte B7-1 also occurs in other clinical conditions characterised by intense proteinuric states. Induction of B7-1 was reported on glomerular podocytes of patients with relapsing minimal change disease (MCD), while it was undetectable in other renal cell subsets [41]. The soluble form of B7-1 was also higher in the urine of relapsing MCD patients compared with individuals with the remitting form of the disease or other conditions, such as systemic lupus erythematosus or glomerulopathies of a different nature [41]. Administration of CTLA4-Ig to B7-1-positive MCD patients produced a dramatic fall in urinary B7-1 and resolution of proteinuria, whereas no therapeutic efficacy was seen in one individual with primary FSGS and three individuals with recurrent FSGS with mild or absent B7-1 expression at the glomerular level [42]. In this respect, there have been reports of problems with detection of podocyte B7-1 and artefacts with immunofluorescence analysis (due in some cases to the use of secondary antibodies directed against goat primary antibodies which can unspecifically cross-react in a proteinuric kidney) [43, 44], highlighting the difficulty of unmasking the dynamic process of upregulation of B7-1 in a timely fashion and the necessity to establish uniformly accepted protocols for its detection. The difficulty of consistently identifying B7-1-positive individuals caused non-linear patterns of CTLA4-Ig therapeutic efficacy (i.e. inconsistent efficacy) in individuals with FSGS. This was mainly due to the absence of negative and/or positive controls in the evaluation of B7-1 expression, and the use of belatacept rather than CTLA4-Ig. Despite targeting both B7-1 and B7-2 (thus some efficacy could be expected in B7-1-positive patients), belatacept has different routes of administration compared with CTLA4-Ig, and greater affinity for B7-2, which is not expressed by podocytes [45-47]. Understanding the limitations of such negative reports may help to avoid misunderstandings related to the efficacy of CTLA4-Ig in proteinuric kidney diseases.

\section{Podocytes and B7-1 in DKD}

We recently reported that podocyte B7-1 is upregulated in the context of DKD [18]. In vitro exposure of podocytes to high glucose (30 mmol// glucose concentration) induced a specific, time-dependent, PI3K-mediated upregulation of podocyte B71 , which was associated with loss of synaptopodin and of activated $\alpha_{3} \beta_{1}$ integrin expression, with disruption of cytoskeletal actin filaments and alteration of cellular motility [18]. Notably, the addition of CTLA4-Ig to the culture medium prevented the detrimental effects of B7-1 and reversed the pathological features described above [18]. High glucose and B7-1 expression also caused podocyte apoptosis and necrosis in vitro, while CTLA4-Ig treatment significantly reduced the rate of controlled and necrotic cell death. CTLA4Ig induced a shift in the pro-/anti-apoptotic balance towards podocyte survival and increased the expression of the antiapoptotic protein Bcl-2, also blunting the activity of the cascade initiator caspase 9 . We subsequently demonstrated that B7-1 upregulation was associated with worsening of kidney damage and with an increase in proteinuria in murine models of type 1 (streptozotocin-induced C57BL/6) and type $2(\mathrm{db} /$ $d b)$ DKD [18]. Treatment with CTLA4-Ig prevented the increase in urinary albumin excretion and histopathological damage to the kidney [18] and, importantly, reversed established proteinuria in streptozotocin-treated C57BL/6 mice. Diabetic mice lacking B7-1 did not benefit from CTLA4-Ig administration and displayed lower urinary protein excretion compared with their diabetic wild-type counterparts when left untreated, confirming that B7-1 is detrimental to kidney function and is required for CTLA4-Ig to deliver therapeutic efficacy. Finally, we demonstrated the expression of B7-1 in biopsies of individuals with type 2 diabetes and DKDrelated glomerular lesions. B7-1 expression was specifically found at the podocyte level, and its degree of upregulation increased with the severity of renal damage, and was accompanied by a parallel loss of synaptopodin expression. Interestingly, we found a significant correlation between the baseline plasma concentration of the soluble form of CD28 [48] and the cumulative incidence of progression to ESRD in a cohort of 200 individuals with type 2 diabetes [18, 49], thus identifying an additional possible soluble mechanism for podocyte B7-1 triggering [18].

\section{Clinical relevance}

As mentioned earlier, CTLA4-Ig (abatacept, Orencia ${ }^{\mathrm{TM}}$, Bristol-Myers Squibb, USA) is a soluble fusion protein that selectively binds B7-1, thus blocking the CD28-B7-1 interaction between $\mathrm{T}$ cells and antigen-presenting cells (Fig. 1a). CTLA4-Ig has been approved by the Food and Drug Administration and European Medicines Agency and is registered for 
the treatment of rheumatoid and juvenile idiopathic arthritis $[36,50]$. CTLA4-Ig is administered intravenously, and its half-life is 17 (range: 12-23) days [51]. The adult dosing regimen for the registered diseases includes a dose ranging from 500 to $1,000 \mathrm{mg}$ depending on body weight and can be administered either by the endovenous or subcutaneous route [52]. The recently approved subcutaneous formulation has comparable efficacy and a similar adverse effect profile to the intravenous formulation [52]. CTLA4-Ig has been administered in more than 4,000 patients in different clinical trials and has been shown to have an optimal profile of efficacy, safety and tolerability [53]. In addition to its well-known use as a biological immunomodulatory agent for the treatment of arthritis, CTLA4-Ig has also been employed in the therapy of recent-onset type 1 diabetes in a double-blind randomised controlled trial, where patients were assigned to receive monthly intravenous doses of either CTLA4-Ig $(10 \mathrm{mg} / \mathrm{kg})$ or placebo for 2 years, to test the efficacy of CTLA4-Ig in delaying the loss of beta cell function [54]. Unfortunately, despite continued administration, the advantage of CTLA4Ig therapy on beta cell function sparing, was less evident after the first 6 months of treatment compared with placebo [54], but the side-effect profile was deemed to be safe (Tables 1 and 2). In agreement with a critical role of podocyte B7-1 in the pathogenesis of kidney disease, CTLA4-Ig has been used for the first time as a specific proteinuria-targeting agent in individuals with B7-1-positive FSGS, successfully reverting urinary protein excretion to normal range thanks to a podocyte-stabilising action on the glomerular basement membrane [37] (Table 1 and Fig. 1b). However, whether those effects in patients result from direct podocyte repair or instead are independent of podocyte B7-1 expression remains to be established, and this represents a necessary step before promoting routine treatment of selected groups of biomarkerpositive patients. Due to the similar pathogenic mechanisms involved in the onset of proteinuria in FSGS and DKD, we now envision the application of CTLA4-Ig therapy in patients with diabetes and initial signs of kidney damage. Patients with normal or mildly reduced renal function (estimated GFR between 45 and $125 \mathrm{ml} \mathrm{min}^{-1} 1.73 \mathrm{~m}^{-2}$ at screening) and albumin excretion rate levels in the high-micro- (90$300 \mathrm{mg} / 24 \mathrm{~h})$ or macro- $(301-2,500 \mathrm{mg} / 24 \mathrm{~h})$ albuminuria range would possibly benefit from a proteinuria-targeting therapy with CTLA4-Ig. The rationale of these inclusion criteria would be to target those patients who still have partially conserved renal function but are at risk of a rapid decline in GFR. A pilot clinical trial investigating whether CTLA4-Ig can control albuminuria in individuals with DKD should be based on the experience collected on the treatment of the diseases for which CTLA4-Ig is approved (i.e. rheumatoid arthritis, FSGS): monthly intravenous boluses $(10 \mathrm{mg} / \mathrm{kg}$ ) or weekly subcutaneous self-administration of CTLA4-Ig $(125 \mathrm{mg})$, with bi-monthly follow-up visits for urinary albumin excretion, GFR and adverse effects occurrence monitoring. Albuminuria would be the ideal candidate biomarker due to the rapid kinetic of change in its concentration and its direct relationship with the anti-proteinuric effect of CTLA4-Ig. Conversely, measurement of the GFR trajectory would require multiple estimations to assess any changes, and the absence of conclusive data on the relationship between GFR and B7-1 expression in type $2 \mathrm{DKD}$, would not justify its use as a primary endpoint. Based on the data collected on the successful treatment of proteinuria in FSGS with CTLA4-Ig, a reduction of urinary albumin excretion rate by $50 \%$ within the first few months of therapy could be expected. It is important to note that, although B7-1 was first discovered in a murine model of lupus nephritis, in a clinical trial studying the effect of CTLA4-Ig in lupus nephritis, treatment did not achieve the primary efficacy measure of the study, which was a composite measure of maintenance of GFR, minimal proteinuria and inactive urinary sediment over the treatment period. Nonetheless, CTLA4-Ig treatment in individuals with lupus nephritis was associated with improved levels of anti-double stranded DNA antibodies, complement $(\mathrm{C} 3$ and $\mathrm{C} 4)$ and a reduction of $20-30 \%$ in the albumin:creatinine ratio. Several limitations may have masked treatment efficacy, including a disproportionate allocation of patients with low urinary protein in the placebo arm and a stringent definition of confirmed complete response (which required the estimated GFR to be equal or higher than $90 \%$ of the reference value), which led to the exclusion of patients who only showed a reduction in proteinuria, thus decreasing the confirmed complete response rates. This is of particular importance considering that the primary effect of CTLA4-Ig in B7-1-positive patients is in fact antiproteinuric [55].

\section{CTLA4-Ig: is it safe?}

Long-term exposure to CTLA4-Ig has been associated with a consistent safe profile during follow-up. Specifically, patients receiving CTLA4-Ig therapy reported minor adverse effects such as headache, nausea, dizziness, cough, back pain, rash and a low incidence rate of more serious side effects (Table 2). Clinical trial and post-marketing surveillance data have indicated an increased risk of opportunistic infections, autoimmune events (the most common being psoriasis) and infusion-site reactions $[56,57]$. Additionally a low incidence of certain malignancies has been reported, consistent with those in a comparable rheumatoid arthritis population $(1.4 \%$ vs $1.1 \%$ for CTLA4-Ig vs placebo, respectively). CTLA4-Ig has also demonstrated low immunogenicity rates, with any occurrence being transient and of a low titre [58]. Given the chronic nature of the diseases for which CTLA4-Ig is approved, these data are of particular relevance to clinicians who use CTLA4-Ig in daily clinical practice and need 
Table 2 Incidence rates of adverse effects events associated with long-term treatment (mean exposure time $27.3 \pm 9.1$ months) with CTLA4-Ig in patients with rheumatoid arthritis

\begin{tabular}{lll}
\hline Event & s.c. CTLA4-Ig $(n=1,879)$ & i.v. CTLA4-Ig $(n=4,149)$ \\
\hline Serious infections & & \\
$\quad$ Pneumonia & $0.36(0.22,0.59)$ & $0.46(0.34,0.59)$ \\
$\quad$ Urinary tract infection & $0.14(0.06,0.32)$ & $0.20(0.13,0.30)$ \\
Gastroenteritis & $0.10(0.04,0.25)$ & $0.12(0.06,0.19)$ \\
Tuberculosis & $0.09(0.04,0.25)$ & $0.07(0.03,0.13)$ \\
Bronchitis & $0.09(0.04,0.25)$ & N/A \\
Lobar pneumonia & $0.07(0.02,0.22)$ & $0.11(0.06,0.18)$ \\
Common malignancies & & \\
Solid organ & $0.69(0.48,0.99)$ & $0.59(0.46,0.75)$ \\
Skin cancer (non-melanoma) & $0.57(0.39,0.86)$ & $0.67(0.53,0.83)$ \\
Basal cell & $0.45(0.29,0.71)$ & $0.48(0.37,0.62)$ \\
Squamous cell & $0.21(0.11,0.41)$ & $0.27(0.18,0.37)$ \\
Melanoma & $0.07(0.02,0.22)$ & $0.03(0.01,0.08)$ \\
Breast & $0.17(0.08,0.35)$ & $0.12(0.06,0.19)$ \\
Lung & $0.12(0.05,0.29)$ & $0.15(0.09,0.23)$ \\
Endometrial/uterine & $0.07(0.02,0.22)$ & $0.03(0.01,0.08)$ \\
Common autoimmune & & $0.57(0.44,0.72)$ \\
Psoriasis & $0.33(0.20,0.56)$ & $0.19(0.12,0.29)$ \\
Sjögren's syndrome & $0.24(0.13,0.44)$ & $0.13(0.08,0.21)$ \\
Erythema nodosum & $0.12(0.05,0.29)$ & $0.12(0.7,0.20)$ \\
Episcleritis & $0.12(0.05,0.29)$ &
\end{tabular}

Results are presented as incidence rate $(95 \% \mathrm{CI})$

Data presented in the table are reproduced from [56] treatments that not only provide a rapid positive response but also minimise long-term safety risks. In this respect, no official studies are available regarding the effects of renal impairment on the pharmacokinetics of CTLA4-Ig; however, because it has a high molecular weight $(\sim 100 \mathrm{kDa})$, CTLA4-Ig is unlikely to be excreted by the kidney, and no strict dose titration is recommended in patients with renal impairment [52], as in the case of patients with DKD.

\section{Discussion}

The evidence that podocytes both upregulate B7-1 in response to LPS or other stimuli, and process/present antigens through MHC class I and II molecules suggests that they may possess functionalities similar to antigen-presenting cells, reacting to innate immune-specific antigens - as in the case of LPS via TLR-4 - or responding in an antigen-specific fashion and priming both $\mathrm{CD}^{+}$and $\mathrm{CD} 8^{+}$T cells [19]. These preliminary findings are further supported by the evidence that podocytes and macrophages share expression of lineage commitment genes (common expression of the transcription factor MafB) as well as other genes [19]. However, due to the interposed basement membrane, the primary function of this response remains unclear. Considering the ultrastructural composition of the podocyte and its contractile nature, another possible interpretation of these events is that, by sensing foreign antigens and by increasing glomerular permeability, the podocyte could facilitate urinary excretion and the removal of toxins or antigens. It should be noted that activation of B7-1 can also be triggered by stimuli of a non-antigenic nature [18, 32, 37]. We recently described a novel feature of renal podocytes - their ability to act as immune-relevant cells of the glomerular microenvironment during stimulation with high glucose concentrations in vitro and hyperglycaemia in vivo [18]. Hyperglycaemia elicits a response that leads to the disengagement of $\alpha_{3} \beta_{1}$ integrin from the glomerular basal membrane and from the cytoskeletal complex, which in turn results in changes in podocyte conformation/motility in vitro and proteinuria in vivo [18], as reported for other stimuli in previous studies [32-34, 37]. The availability of a clinical-grade drug capable of targeting B7-1 makes this field of investigation particularly attractive (Table 1 ). The evidence that CTLA4Ig effectively binds B7-1 at the podocyte level when administered in vitro, and that its positive effects take place independently of the immune system - previously reported to be involved in DKD progression [59] — led us to hypothesise that CTLA4-Ig may exert a novel anti-proteinuric function in B71-positive kidney diseases [18, 37] (Fig. 1b). Although the signalling that underlies podocyte B7-1 activation and its 
proteinuria-triggering role has recently been defined, the mechanism of CTLA4-Ig action on B7-1 has yet to be revealed. CTLA4-Ig could either sterically block the interaction of B7-1 with $\alpha_{3} \beta_{1}$ integrin or could alter the tertiary structure of B7-1, impeding the translocation of its cytoplasmic tail towards integrin tails. Another potential mechanism of action involves competition of CTLA4-Ig with soluble CD28 for binding to B7-1, the latter being associated with a worse outcome in ESRD patients [18]. This last possibility could be further explored given the availability of the fusion protein CD28-Ig for both in vitro and in vivo studies. Importantly, by specifically targeting proteinuria - a demonstrated determinant of renal function decline [60] C CTLA4-Ig would have a different therapeutic action from the anti-hypertensive and glucose-lowering approaches attempted thus far. In this respect, a strategy to demonstrate that CTLA4-Ig has therapeutic efficacy in podocyte B7-1-positive patients would be particularly helpful. Since a kidney biopsy-based strategy to study renal expression of B7-1 is not feasible, great efforts should be made to identify the subgroup of B7-1-positive individuals who are more likely to benefit from CTLA4-Ig treatment. As in the case of rheumatoid arthritis, where circulating B7-1 protein levels were found to predict the response to CTLA4-Ig [61], a peripheral proxy (e.g. plasma and/or urine) of podocyte B7-1 expression would not only represent an easy method of selecting those patients to be treated but would also provide a practical means of following up these individuals and assessing the efficacy of CTLA4-Ig administration over time. Of the candidate factors affecting podocyte function in DKD [62], it would be particularly interesting to investigate whether, as suggested by our data [18], soluble CD28 per se represents a circulating molecule that causes B7-1-dependent signalling in DKD. If so, the next step would be to determine whether circulating levels of soluble CD28 are modified by CTLA4Ig treatment (as a result of the competitive binding of CTLA4-Ig to podocyte B7-1), making CD28 a suitable peripheral marker of treatment efficacy.

Finally, not all individuals with diabetes and kidney damage follow the albuminuric pathway to renal impairment, and the mechanism(s) of DKD progression in the absence of proteinuria is not clear. One could speculate that in those individuals with low microalbuminuria who are more susceptible to tubular damage (due to the presence of albumin at this site) as the disease progresses there is extensive tubular damage, detectable as a drop in GFR, rather than glomerular injury and increasing albuminuria. In this case, targeting B7-1 with CTLA4-Ig could limit the worsening of albuminuria and control this pathological pathway. At the other end of the spectrum, GFR decline and albuminuria can be viewed as two different pathways that might or might not converge in an individual patient, and for which different therapeutic approaches should be considered.
In conclusion, recent evidence has identified the upregulation of B7-1 as a novel mechanism of kidney damage in DKD. Interestingly, CTLA4-Ig, which is clinically available for the treatment of inflammatory and immunological disorders and which targets B7-1, has been shown to effectively reduce proteinuria in B7-1-positive FSGS patients and therefore can be hypothesised to have beneficial effects on DKD as well. However, rigorous clinical trials must be performed to test the potential therapeutic efficacy and the safety of CTLA4-Ig in the context of B7-1-positive diabetic and non-diabetic kidney disease.

Funding RB is the recipient of an AST Genentech/Novartis Clinical Science Fellowship grant, a JDRF Post-Doctoral Fellowship grant and an 'AMD-SID Pasquale di Coste' Award. PF is the recipient of an American Heart Association Grant-In-Aid; Italian Ministry of Health grant RF2010-2303119 and RF-2010-2314794. AF is supported by NIH grants R01-DK090316, U24-DK076169, UL1-TR000460, U54-DK083912 and by the Katz Drug Discovery Center.

Duality of interest The authors have no relevant conflicts of interest to disclose. AF is the inventor of a patent application entitled: Assays, Methods and kits for prediction of renal disease and personalized treatment strategies. Application Number PCT/US11/56272. AF is CSO and Vice-President of L\&F Health LLC, a company aimed at the development of personalised treatment strategies for patients with proteinuric kidney disease. AF has signed a research agreement with Hoffmann-La Roche, Abbvie, Mesoblast and Bristol-Myers Squibb. PF has signed research agreements with Dompè Pharmaceuticals and Fate Therapeutics that are not related to the topic of this article.

Contribution statement $\mathrm{RB}$ and $\mathrm{AF}$ designed and drafted the article. $\mathrm{RB}$ and $\mathrm{AF}$ acquired, analysed and interpreted data. $\mathrm{AD}$ and $\mathrm{PF}$ drafted and revised the article. All authors have approved the version to be published.

\section{References}

1. Gross JL, de Azevedo MJ, Silveiro SP, Canani LH, Caramori ML, Zelmanovitz T (2005) Diabetic nephropathy: diagnosis, prevention, and treatment. Diabetes Care 28:164-176

2. Bassi R, Trevisani A, Tezza S et al (2012) Regenerative therapies for diabetic microangiopathy. Exp Diabetes Res 2012:916560

3. Go AS, Chertow GM, Fan D, McCulloch CE, Hsu CY (2004) Chronic kidney disease and the risks of death, cardiovascular events, and hospitalization. N Engl J Med 351:1296-1305

4. National Kidney Foundation (2012) KDOQI clinical practice guideline for diabetes and CKD: 2012 update. Am J Kidney Dis 60:850-886

5. Molitch ME, Steffes M, Sun W et al (2010) Development and progression of renal insufficiency with and without albuminuria in adults with type 1 diabetes in the diabetes control and complications trial and the epidemiology of diabetes interventions and complications study. Diabetes Care 33:1536-1543

6. Caramori ML, Fioretto P, Mauer M (2000) The need for early predictors of diabetic nephropathy risk: is albumin excretion rate sufficient? Diabetes 49:1399-1408 
7. MacIsaac RJ, Tsalamandris C, Panagiotopoulos S, Smith TJ, McNeil KJ, Jerums G (2004) Nonalbuminuric renal insufficiency in type 2 diabetes. Diabetes Care 27:195-200

8. Krolewski AS, Niewczas MA, Skupien J et al (2014) Early progressive renal decline precedes the onset of microalbuminuria and its progression to macroalbuminuria. Diabetes Care 37:226-234

9. Valmadrid CT, Klein R, Moss SE, Klein BE (2000) The risk of cardiovascular disease mortality associated with microalbuminuria and gross proteinuria in persons with older-onset diabetes mellitus. Arch Intern Med 160:1093-1100

10. Afkarian M, Sachs MC, Kestenbaum B et al (2013) Kidney disease and increased mortality risk in type 2 diabetes. J Am Soc Nephrol 24:302-308

11. Jerums G, Panagiotopoulos S, Premaratne E, MacIsaac RJ (2009) Integrating albuminuria and GFR in the assessment of diabetic nephropathy. Nat Rev Nephrol 5:397-406

12. Nathan DM, Buse JB, Davidson MB et al (2009) Medical management of hyperglycaemia in type 2 diabetes: a consensus algorithm for the initiation and adjustment of therapy: a consensus statement of the American Diabetes Association and the European Association for the Study of Diabetes. Diabetologia 52:17-30

13. de Boer IH, Sun W, Cleary PA et al (2011) Intensive diabetes therapy and glomerular filtration rate in type 1 diabetes. N Engl J Med 365:2366-2376

14. Fullerton B, Jeitler K, Seitz M, Horvath K, Berghold A, Siebenhofer A (2014) Intensive glucose control versus conventional glucose control for type 1 diabetes mellitus. Cochrane Database Syst Rev, Issue 2, Art. no.: CD009122. doi:10.1002/14651858. CD009122.pub2

15. Lewis EJ, Hunsicker LG, Bain RP, Rohde RD (1993) The effect of angiotensin-converting-enzyme inhibition on diabetic nephropathy. The Collaborative Study Group. N Engl J Med 329:1456-1462

16. Taal MW, Brenner BM (2000) Renoprotective benefits of RAS inhibition: from ACEI to angiotensin II antagonists. Kidney Int 57:1803-1817

17. Mundel P, Shankland SJ (2002) Podocyte biology and response to injury. J Am Soc Nephrol 13:3005-3015

18. Fiorina P, Vergani A, Bassi R et al (2014) Role of podocyte B7-1 in diabetic nephropathy. J Am Soc Nephrol 25:1415-1429

19. Goldwich A, Burkard M, Olke M et al (2013) Podocytes are nonhematopoietic professional antigen-presenting cells. J Am Soc Nephrol 24:906-916

20. Banas MC, Banas B, Hudkins KL et al (2008) TLR4 links podocytes with the innate immune system to mediate glomerular injury. J Am Soc Nephrol 19:704-713

21. Reiser J, von Gersdorff G, Loos M et al (2004) Induction of B7-1 in podocytes is associated with nephrotic syndrome. J Clin Invest 113:1390-1397

22. Niemir ZI, Stein H, Dworacki G et al (1997) Podocytes are the major source of IL-1 alpha and IL-1 beta in human glomerulonephritides. Kidney Int 52:393-403

23. Chow FY, Nikolic-Paterson DJ, Ozols E, Atkins RC, Rollin BJ, Tesch GH (2006) Monocyte chemoattractant protein-1 promotes the development of diabetic renal injury in streptozotocin-treated mice. Kidney Int 69:73-80

24. Huber TB, Reinhardt HC, Exner M et al (2002) Expression of functional CCR and CXCR chemokine receptors in podocytes. J Immunol 168:6244-6252

25. Kuravi SJ, McGettrick HM, Satchell SC et al (2014) Podocytes regulate neutrophil recruitment by glomerular endothelial cells via IL-6-mediated crosstalk. J Immunol 193:234-243

26. Nolasco FE, Cameron JS, Hartley B, Coelho RA, Hildredth G, Reuben R (1987) Abnormal podocyte CR-1 expression in glomerular diseases: association with glomerular cell proliferation and monocyte infiltration. Nephrol Dial Transplant 2:304-312
27. Abe K, Miyazaki M, Koji T et al (2001) Enhanced expression of complement $\mathrm{C} 5 \mathrm{a}$ receptor mRNA in human diseased kidney assessed by in situ hybridization. Kidney Int 60:137-146

28. Delville M, Sigdel TK, Wei C et al (2014) A circulating antibody panel for pretransplant prediction of FSGS recurrence after kidney transplantation. Sci Transl Med 6:256ra136

29. Doria A, Niewczas MA, Fiorina P (2012) Can existing drugs approved for other indications retard renal function decline in patients with type 1 diabetes and nephropathy? Semin Nephrol 32:437-444

30. Sayegh MH, Turka LA (1998) The role of T-cell costimulatory activation pathways in transplant rejection. N Engl J Med 338: 1813-1821

31. Chandraker A, Azuma H, Nadeau K et al (1998) Late blockade of T cell costimulation interrupts progression of experimental chronic allograft rejection. J Clin Invest 101:2309-2318

32. Agrawal S, Guess AJ, Chanley MA, Smoyer WE (2014) Albumininduced podocyte injury and protection are associated with regulation of COX-2. Kidney Int 86:1150-1160

33. Zhang J, Gu C, Lawrence DA, Cheung AK, Huang Y (2014) A plasminogen activator inhibitor type 1 mutant retards diabetic nephropathy in $d b / d b$ mice by protecting podocytes. Exp Physiol 99: 802-815

34. Chang JM, Hwang DY, Chen SC et al (2013) B7-1 expression regulates the hypoxia-driven cytoskeleton rearrangement in glomerular podocytes. Am J Physiol Ren Physiol 304:F127-F136

35. Ishimoto T, Shimada M, Gabriela G et al (2013) Toll-like receptor 3 ligand, polyIC, induces proteinuria and glomerular CD80, and increases urinary CD80 in mice. Nephrol Dial Transplant 28:14391446

36. Genovese MC, Becker JC, Schiff M et al (2005) Abatacept for rheumatoid arthritis refractory to tumor necrosis factor alpha inhibition. N Engl J Med 353:1114-1123

37. Yu CC, Fornoni A, Weins A et al (2013) Abatacept in B7-1-positive proteinuric kidney disease. N Engl J Med 369:2416-2423

38. Fornoni A (2010) Proteinuria, the podocyte, and insulin resistance. N Engl J Med 363:2068-2069

39. Tian X, Kim JJ, Monkley SM et al (2014) Podocyte-associated talin1 is critical for glomerular filtration barrier maintenance. J Clin Invest 124:1098-1113

40. Fornoni A, Sageshima J, Wei C et al (2011) Rituximab targets podocytes in recurrent focal segmental glomerulosclerosis. Sci Transl Med 3:85ra46

41. Garin EH, Diaz LN, Mu W et al (2009) Urinary CD80 excretion increases in idiopathic minimal-change disease. J Am Soc Nephrol 20:260-266

42. Garin EH, Reiser J, Cara-Fuentes G et al (2015) Case series: CTLA4-IgG1 therapy in minimal change disease and focal segmental glomerulosclerosis. Pediatr Nephrol 30:469-477

43. Benigni A, Gagliardini E, Remuzzi G (2014) Abatacept in B7-1positive proteinuric kidney disease. N Engl J Med 370:1261-1263

44. Larsen CP, Messias NC, Walker PD (2014) B7-1 immunostaining in proteinuric kidney disease. Am J Kidney Dis 64:1001-1003

45. Alachkar N, Carter-Monroe N, Reiser J (2014) Abatacept in B7-1positive proteinuric kidney disease. N Engl J Med 370:1263-1264

46. Grellier J, Del Bello A, Milongo D, Guilbeau-Frugier C, Rostaing L, Kamar N (2015) Belatacept in recurrent focal segmental glomerulosclerosis after kidney transplantation. Transpl Int 28:1109-1110

47. Martin ST, Tichy EM, Gabardi S (2011) Belatacept: a novel biologic for maintenance immunosuppression after renal transplantation. Pharmacotherapy 31:394-407

48. Orabona C, Grohmann U, Belladonna ML et al (2004) CD28 induces immunostimulatory signals in dendritic cells via CD80 and CD86. Nat Immunol 5:1134-1142 
49. Niewczas MA, Gohda T, Skupien J et al (2012) Circulating TNF receptors 1 and 2 predict ESRD in type 2 diabetes. J Am Soc Nephrol 23:507-515

50. Ruperto N, Lovell DJ, Quartier P et al (2008) Abatacept in children with juvenile idiopathic arthritis: a randomised, double-blind, placebo-controlled withdrawal trial. Lancet 372:383-391

51. Ma Y, Lin BR, Lin B et al (2009) Pharmacokinetics of CTLA4Ig fusion protein in healthy volunteers and patients with rheumatoid arthritis. Acta Pharmacol Sin 30:364-371

52. Reynolds J, Shojania K, Marra CA (2007) Abatacept: a novel treatment for moderate-to-severe rheumatoid arthritis. Pharmacotherapy 27:1693-1701

53. Atzeni F, Sarzi-Puttini P, Mutti A, Bugatti S, Cavagna L, Caporali R (2013) Long-term safety of abatacept in patients with rheumatoid arthritis. Autoimmun Rev 12:1115-1117

54. Orban T, Bundy B, Becker DJ et al (2011) Co-stimulation modulation with abatacept in patients with recent-onset type 1 diabetes: a randomised, double-blind, placebo-controlled trial. Lancet 378:412-419

55. Furie R, Nicholls K, Cheng TT et al (2014) Efficacy and safety of abatacept in lupus nephritis: a twelve-month, randomized, doubleblind study. Arthritis Rheum 66:379-389

56. Alten R, Kaine J, Keystone E, Nash P, Delaet I, Genovese MC (2014) Long-term safety of subcutaneous abatacept in rheumatoid arthritis: integrated analysis of clinical trial data representing more than four years of treatment. Arthritis Rheum 66:1987-1997

57. Weinblatt ME, Moreland LW, Westhovens R et al (2013) Safety of abatacept administered intravenously in treatment of rheumatoid arthritis: integrated analyses of up to 8 years of treatment from the abatacept clinical trial program. J Rheumatol 40:787-797

58. Nash P, Nayiager S, Genovese MC et al (2013) Immunogenicity, safety, and efficacy of abatacept administered subcutaneously with or without background methotrexate in patients with rheumatoid arthritis: results from a phase III, international, multicenter, parallel-arm, open-label study. Arthritis Care Res (Hoboken) 65:718728

59. D'Addio F, Trevisani A, Ben Nasr M et al (2014) Harnessing the immunological properties of stem cells as a therapeutic option for diabetic nephropathy. Acta Diabetol 51:897-904

60. Abbate M, Zoja C, Remuzzi G (2006) How does proteinuria cause progressive renal damage? J Am Soc Nephrol 17:2974-2984

61. Scarsi M, Ziglioli T, Airo P (2011) Baseline numbers of circulating CD28-negative $T$ cells may predict clinical response to abatacept in patients with rheumatoid arthritis. J Rheumatol 38:2105-2111

62. Diez-Sampedro A, Lenz O, Fornoni A (2011) Podocytopathy in diabetes: a metabolic and endocrine disorder. Am J Kidney Dis 58:637-646 